\title{
Quaternion-Based Robust Satellite Attitude Tracking Control
}

\author{
J. Dasdemir
}

\begin{abstract}
In this paper, a nonlinear robust quaternion-based controller is developed to address the three-axis attitude tracking control problem of rigid spacecraft in presence of parametric uncertainties, unknown external disturbances and sensor noise. As a first step, a robust controller is designed that compensates parametric uncertainty and disturbance effects. The robust controller then reformulated to deal also with sensor noise. Singularity free unit quaternions are used to represent the attitude of the satellite in three-dimensional space. The Lyapunov-based stability analysis is applied to prove that a uniformly ultimately bounded tracking result is achieved. Simulation results are presented to illustrate the feasibility of the proposed control strategy.
\end{abstract}

Index Terms - Attitude tracking, unit quaternion, robust control, sensor noise.

\section{INTRODUCTION}

$\mathrm{T}$ HE attitude tracking control problem of rigid bodies received much attention for decades due to its application areas ranging from robot manipulators to satellites and still continuous to be an active research area [1-3]. Especially, in applications such as satellite surveillance and communication, a considerable amount of effort has been devoted to solve both stabilization and tracking problems. The challenging nature of the attitude tracking control problem arises not only from the nonlinearity of the dynamics but also the representation of the attitude kinematics. There are several kinematic parameterizations for orientation angles [4]: Three-parameter representations such as Euler angles, Rodriguez parameters and modified Rodrigues parameters, limited because of kinematic singularities and non-singular, four-parameter representation by unit quaternions with a constraint equation. The control problem can be further complicated in presence of parametric uncertainties, unknown disturbances and sensor noise simultaneously.

The attitude control problem of a spacecraft in the presence of disturbance and/or uncertainties has been extensively studied. Many different control strategies including, adaptive control [5-6], sliding mode control [7-8] and robust control methods [9-10] were proposed in the literature and all these methods have their own advantages as well as disadvantages. Sliding mode control is a widely preferred control method due to its simple and inherently robust structure.

J. DASDEMIR is with Department of Control and Automation Eng., Yildiz Technical University, Istanbul, Turkey, (e-mail: janset@yildiz.edu.tr) (iD)

Manuscript received September 19, 2017; accepted January 11, 2018. DOI: $10.17694 /$ bajece.402013
However, sliding mode control might excite the high frequency unmodeled dynamics in practical applications [11]. Thus, it can lead to control performance deterioration. A typical adaptive control mechanism is the combination of on-line estimation and control and it has limitations that if uncertain parameters enter the system model in complicated ways, it might be problematic to obtain continuously parameterized control structures and they are also not robust against external disturbances [12-13]. Robust controllers exhibit satisfactory performance against different types of disturbances but they might have large torque demands. Exponential convergence rate with infinite settling time is also a drawback of conventional robust and sliding mode control techniques [14]. In order to eliminate these shortcomings, there are several works on designing finite time control structures mostly based on terminal sliding mode control. However, most of the proposed methods present either slow convergence speed, if state is away from equilibrium, or posses chattering phenomena due to discontinuities [15-16].

All the aforementioned control methods have strength against model uncertainties and/or disturbances. However, none of these papers have considered sensor noise effects. Attitude tracking control of a rigid spacecraft in presence of parametric uncertainties, unknown disturbances and sensor noise is an important problem and should be considered together in control development, in particular for real-time implementations. Performance of a control algorithm also depends on the quality of the available measurements and online computation of the algorithm. For applications with demanding sampling times or limited computational recourses, this on-line computation requirement can be problematic [17]. If the control law is also contaminated by sensor noise, performance degradation is inevitable. Moreover, low cost, small satellites considered in this study require less energy consumption and do not have sufficient onboard resources to implement complex algorithms [18]. The onboard hardware is usually very limited. Hence, complicated control structures may not perform well particularly in presence of sensor noise. In this work, to overcome this implementation issues, the significant part of the control algorithm formulated without dependence on measurement values. Instead, the predefined desired values have been utilized. Hence, computation burden, sensor contaminations and control energy requirement are relatively reduced.

In this paper, a new robust attitude tracking control method that takes into account the simultaneous effects of parametric uncertainties, external disturbances and measurement noise has been proposed. Firstly, a transformation, introduced in [19] is used for the open-loop tracking error dynamics. As a first step, a typical robust controller is designed to compensate the 
adverse effects of uncertainties in inertia matrix and external disturbances. Then, ultimate boundedness result is obtained through Lyapunov-type stability analysis. Subsequently, to obtain a better performance in the presence of sensor noise, the former control algorithm is exploited to design the new control structure and it is shown that the proposed control method ensures uniformly ultimately bounded attitude tracking.

The paper is organized as follows: Section II provides the dynamic and kinematic model of the satellite. The problem statement and the open-loop error system dynamics are introduced in Section III. In Section IV and V, both standard robust control and the newly proposed control developments are presented. Numerical results are shown in Section VI. Finally, Section VII presents some concluding remarks.

\section{MODEL FORMULATION}

\section{A. Dynamic Model}

Motivated by the need to obtain the dynamic and kinematic model of the rigid spacecraft, two frames denoted by $I=$ $\left\{x_{I}, y_{I}, z_{I}\right\}$ and $\mathcal{B}=\left\{x_{\mathcal{B}}, y_{\mathcal{B}}, z_{\mathcal{B}}\right\}$ are utilized. Let $I$ be the inertial reference frame and the origin of this orthogonal frame is located in the center of the earth. The other frame $\mathcal{B}$ is the bodyfixed frame attached to the center of mass of the satellite. There are also reaction wheels, as actuator mechanisms, located on each axis of the body-fixed frame to control the attitude of the rigid spacecraft. The dynamic model of the satellite can be expressed as follows [20]:

$$
J \dot{\omega}=-\omega^{\times} J \omega+\tau+\tau_{d}
$$

where $J \in \mathbb{R}^{3 \times 3}$ is positive-definite, constant, symmetric inertia matrix, $\omega(t) \in \mathbb{R}^{3}$ is the angular velocity of the body-fixed frame with respect to the inertial reference frame. $\tau(t) \in \mathbb{R}^{3}$ is the torque input control vector and $\tau_{d} \in \mathbb{R}^{3}$ is a bounded disturbance vector, and the notation $\zeta^{\times}, \forall \zeta=\left[\begin{array}{lll}\zeta_{1} & \zeta_{2} & \zeta_{3}\end{array}\right]^{T}$ represents the skew-symmetric matrix:

$$
\zeta^{\times}=\left[\begin{array}{ccc}
0 & -\zeta_{3} & \zeta_{2} \\
\zeta_{3} & 0 & -\zeta_{1} \\
-\zeta_{2} & \zeta_{1} & 0
\end{array}\right]
$$

Property 1: The inertia matrix can be lower and upper bounded by the following inequalities:

$$
j_{1}\|\xi\|^{2} \leq \xi^{T} J \xi \leq j_{2}\|\xi\|^{2} \quad \forall \xi \in \mathbb{R}^{3}
$$

Where $j_{1}, j_{2} \in \mathbb{R}$ are some positive constants, and $\|\cdot\|$ denotes the standard Euclidean norm.

Remark 1: The disturbance vector $\tau_{d}(t)=$ $\left[\tau_{d 1}(t), \tau_{d 2}(t), \tau_{d 3}(t)\right]^{T}$ can be upper bounded as follows:

$$
\left\|\tau_{d}\right\| \leq \xi_{t d}
$$

\section{B. Kinematic Model}

In this this work, kinematics of the satellite will be described using unit quaternions, due to their nonsingular and computationally effective representation. According to the Euler's Theorem, any rotation matrix can be defined uniquely by a rotation angle $\vartheta(t) \in \mathbb{R}$ about an appropriate unit vector $k(t) \in \mathbb{R}^{3}$. Thus, based on a given $(\vartheta, k) \in \mathbb{R}^{4}$, an alternative parametrization of the attitude can be obtained by fourparameter unit quaternions as follows [20]:

$$
\begin{gathered}
q_{0}(t) \triangleq \cos \left(\frac{1}{2} \vartheta\right) \\
q_{v}(t) \triangleq k \sin \left(\frac{1}{2} \vartheta\right)
\end{gathered}
$$

In Equations (5) and (6), $q(t)=\left\{q_{0}(t), q_{v}(t)\right\} \in \mathbb{R} \times \mathbb{R}^{3}$ denotes the unit quaternion defining the orientation of the bodyfixed frame $\mathcal{B}$ with respect to the inertial frame I, subject to the constraint

$$
q_{v}^{T} q_{v}+q_{0}^{2}=1
$$

The rotation matrix $R\left(q_{0}, q_{v}\right) \in S O(3)$ that brings $I$ onto $\mathcal{B}$ can be calculated as

$$
R=\left(q_{0}^{2}-q_{v}^{T} q_{v}\right) I_{3}+2 q_{v} q_{v}^{T}-2 q_{0} q_{v}^{\times}
$$

where $I_{3} \in \mathbb{R}^{3 \times 3}$ represents $3 \times 3$ identity matrix. Hence, the differential equation describing the attitude kinematics of the satellite can be expressed by using unit quaternions as follows [21]:

$$
\begin{gathered}
\dot{q}_{0}=-\frac{1}{2} q_{v}^{T} \omega \\
\dot{q}_{v}=\frac{1}{2}\left(q_{v}^{\times} \omega+q_{0} \omega\right)
\end{gathered}
$$

Equations (9) and (10) can be modified as

$$
\dot{q}=\frac{1}{2} \Sigma(q) \omega
$$

where $\Sigma(q)=\left[\Sigma_{0}^{T}, \Sigma_{v}^{T}\right]^{T} \in \mathbb{R}^{4 \times 3}$ with $\Sigma_{0}=-q_{v}^{T}$ and $\Sigma_{v}=$ $q_{v}^{\times}+q_{0} I_{3}$ respectively. The angular velocity of the body-fixed frame with respect to the inertial frame expressed in the bodyfixed frame can be obtained according to Equations (9) and (10) in the following form:

$$
\omega=2\left(q_{0} \dot{q}_{v}-q_{v} \dot{q}_{0}\right)-2 q_{v}^{\times} \dot{q}_{v}
$$

\section{PROBLEM STATEMENT AND QUATERNION ERROR DYNAMICS}

In order to express the desired attitude of the satellite, another body-fixed reference frame $\mathcal{B}_{d}=\left\{x_{\mathcal{B} d}, y_{\mathcal{B} d}, z_{\mathcal{B} d}\right\}$ is defined. The orientation of this frame with respect to the inertial frame can be defined by the desired unit quaternion $q_{d}(t) \triangleq$ $\left\{q_{0 d}(t), q_{v d}(t)\right\} \in \mathbb{R} \times \mathbb{R}^{3}$ with a similar constraint given in Equation (7):

$$
q_{v d}^{T} q_{v d}+q_{0 d}^{2}=1
$$

The rotation matrix that brings inertial frame $I$ onto the desired reference body-fixed frame $\mathcal{B}_{d}$ can be calculated as

$$
R_{d}=\left(q_{0 d}^{2}-q_{v d}^{T} q_{v d}\right) I_{3}+2 q_{v d} q_{v d}^{T}-2 q_{0 d} q_{v d}^{\times}
$$


The relation between the desired unit quaternion and the angular velocity of the desired body-fixed reference frame with respect to the inertial frame expressed in desired frame $\omega_{d}(t) \in$ $\mathbb{R}^{3}$ can be stated as follows:

$$
\begin{gathered}
\dot{q}_{0 d}=-\frac{1}{2} q_{v d}^{T} \omega_{d} \\
\dot{q}_{v}=\frac{1}{2}\left(q_{v}^{\times} \omega+q_{0} \omega\right)
\end{gathered}
$$

Through the help of $\Sigma_{d}(q)=\left[\Sigma_{0 d}^{T}, \Sigma_{v d}^{T}\right]^{T} \in \mathbb{R}^{4 \times 3}$ with $\Sigma_{0 d}=$ $-q_{v d}^{T}$ and $\Sigma_{v d}=q_{v d}^{\times}+q_{0 d} I_{3}$ respectively, Equations (15-16) can be written in a compact form as

$$
\dot{q}_{d}=\frac{1}{2} \Sigma_{d}(q) \omega_{d}
$$

Utilizing Equations (15-16), the desired angular velocity can be obtained as:

$$
\omega_{d}=2\left(q_{0 d} \dot{q}_{v d}-q_{v d} \dot{q}_{0 d}\right)-2 q_{v d}^{\times} \dot{q}_{v d}
$$

In order to achieve the control objective, the attitude of the body-fixed frame $\mathcal{B}$ should track the desired body-fixed frame $\mathcal{B}_{d}$. To describe this objective, an attitude tracking error term $\tilde{R}\left(e_{0}, e_{v}\right) \in \mathbb{R}^{3 \times 3}$, defining the mismatch, stated in the following as performance criteria

$$
\tilde{R} \triangleq R R^{T}=\left(e_{0}^{2}-e_{v}^{T} e_{v}\right) I_{3}+2 e_{v} e_{v}^{T}-2 e_{0} e_{v}^{\times}
$$

where the quaternion tracking error $e(t) \triangleq\left\{e_{0}(t), e_{v}(t)\right\} \in$ $\mathbb{R} \times \mathbb{R}^{3}$ can be represented explicitly as follows with a similar constraint given in Equation (7).

$$
\begin{gathered}
e_{0}=q_{0} q_{0 d}+q_{v}^{T} q_{v d} \\
e_{v}=q_{0 d} q_{v}-q_{0} q_{v d}+q_{v}^{\times} q_{v d} \\
e_{v}^{T} e_{v}+e_{0}^{2}=1
\end{gathered}
$$

Note that according to the Equation (19), the control goal will be achieved as the mismatch term converges to the identity matrix as follows

$$
\lim _{t \rightarrow \infty} \tilde{R} \rightarrow I_{3}
$$

It is obvious that Equation (23) will be guaranteed if the following condition is satisfied

$$
\left\|e_{v}(t)\right\| \rightarrow 0 \Longrightarrow\left|e_{0}\right| \rightarrow 1
$$

and according to Equation (22)

$$
0 \leq\left\|e_{v}(t)\right\| \leq 1, \quad 0 \leq\left|e_{0}(t)\right| \leq 1
$$

The angular velocity tracking error can also be defined by the use of Equation (19) in the following form

$$
\widetilde{\omega}=\omega-\tilde{R} \omega_{d}
$$

Based on the previous formulations, specifically, using Equation (1), (9-10), (15-16), (20-21) and (26) The open loop tracking error dynamics of the satellite to be controlled can be obtained as follows

$$
\begin{gathered}
J \dot{\widetilde{\omega}}=-\left(\widetilde{\omega}+\tilde{R} \omega_{d}\right)^{\times} J\left(\widetilde{\omega}+\widetilde{R} \omega_{d}\right) \\
+J\left(\widetilde{\omega} \times \tilde{R} \omega_{d}-\tilde{R} \dot{\omega}_{d}\right)+\tau+\tau_{d} \\
\dot{e}_{v}=\frac{1}{2}\left(e_{v}^{\times}+e_{0} I_{3}\right) \widetilde{\omega} \\
\dot{e}_{0}=-\frac{1}{2} e_{v}^{T} \widetilde{\omega}
\end{gathered}
$$

Remark 2: It is supposed that both $q_{0 d}(t)$ and $q_{v d}(t)$ and their first two time derivatives are bounded. Thus, $\omega_{d}(t)$ and its derivative are bounded for all time.

Equation (28) can be modified with the use of $T\left(e_{0}, e_{v}\right) \in \mathbb{R}^{3 \times 3}$ as defined below

$$
\begin{gathered}
T \triangleq e_{v}^{\times}+e_{0} I_{3} \\
\dot{e}_{v}=\frac{1}{2} T \widetilde{\omega}
\end{gathered}
$$

Taking the time derivative of (31) and premultiplying both sides of the resulting equation by $T^{-T} J T^{-1}$

$$
J^{*} \ddot{e}_{v}=\frac{1}{2} J^{*} \dot{T} \widetilde{\omega}+\frac{1}{2} P^{T} J \dot{\widetilde{\omega}}
$$

can be obtained where $J^{*}\left(e_{v}, e_{0}\right) \in \mathbb{R}^{3 \times 3}$ and $P\left(e_{v}, e_{0}\right) \in \mathbb{R}^{3 \times 3}$ are defined as follows:

$$
\begin{gathered}
J^{*} \ddot{e}_{v}=\frac{1}{2} J^{*} \dot{T} \widetilde{\omega}+\frac{1}{2} P^{T} J \dot{\widetilde{\omega}} \\
J^{*} \triangleq P^{T} J P
\end{gathered}
$$

After substituting (27) into (32), the following open loop error dynamics can be attained

$$
J^{*} \ddot{e}_{v}+C^{*} \dot{e}_{v}+N^{*}-\tau_{d}^{*}=\tau^{*}
$$

where the new terms $\tau^{*} \in \mathbb{R}^{3}, \tau_{d}^{*} \in \mathbb{R}^{3}, C^{*}\left(e_{v}, e_{0}, \dot{e}_{v}\right) \in \mathbb{R}^{3 \times 3}$ and $N^{*}\left(e_{v}, e_{0}, \dot{e}_{v}, \omega_{d}, \dot{\omega}_{d}\right) \in \mathbb{R}^{3}$ are defined as

$$
\begin{gathered}
\tau^{*} \triangleq \frac{1}{2} P^{T} \tau \\
\tau_{d}^{*} \triangleq \frac{1}{2} P^{T} \tau_{d} \\
C^{*} \triangleq-J^{*} \dot{P}^{-1} P-2 P^{T}\left(J P \dot{e}_{v}\right)^{\times} P
\end{gathered}
$$




$$
\begin{aligned}
N^{*} \triangleq P^{T}\left[\left(P \dot{e}_{v}\right)^{\times} J \tilde{R} \omega_{d}\right]+P^{T}\left[\left(\tilde{R} \omega_{d}\right)^{\times} P \dot{e}_{v}\right] \\
-\frac{1}{2} P^{T} J\left[\left(2 P \dot{e}_{v}\right)^{\times} \tilde{R} \omega_{d}-\tilde{R} \dot{\omega}_{d}\right] \\
+\frac{1}{2} P^{T}\left[\left(\tilde{R} \omega_{d}\right)^{\times} J \tilde{R} \omega_{d}\right]
\end{aligned}
$$

The following property will be utilized in the subsequent controller design and analysis sections

Property 2: The inertia and the centripetal- Coriolis matrices satisfy the following skew-symmetric property

$$
\xi^{T}\left(\frac{1}{2} j^{*}-C^{*}\right) \xi=0 \quad \forall \xi \in \mathbb{R}^{3}
$$

Remark3: Note that the Jacobian matrix in Equation (30) can be invertible, if the following condition is satisfied

$$
\operatorname{det}(T)=e_{0}(t) \neq 0, \quad \forall t \in[0, \infty)
$$

To guarantee Equation (41), initial conditions should be restricted that $e_{0}(0) \neq 0$ and the proposed control method should also provide $e_{0}(t) \neq 0$. It is obvious from Equation (24) and $(20)$ that the desired trajectory can be initialized to ensure that $e_{0}(0) \neq 0$.

\section{ROBUST CONTROL DEVELOPMENT}

In this section, the objective is to design a robust attitudetracking controller for the open-loop error dynamics given in Equation (35) under the assumption that the satellite inertia matrix and disturbance vector are unknown. To quantify the mismatch, the parameter estimation error, $\tilde{\theta} \in \mathbb{R}^{6}$ and the disturbance estimation error, $\tilde{\tau}_{d}^{*} \in \mathbb{R}^{3}$ are defined as

$$
\begin{gathered}
\tilde{\theta} \triangleq \theta-\hat{\theta} \\
\tilde{\tau}_{d}^{*}=\tau_{d}^{*}-\hat{\tau}_{d}^{*}
\end{gathered}
$$

where $\hat{\theta}$ and $\hat{\tau}_{d}^{*}$ are the best guess-estimates of the unknown vector of inertia parameters $\theta \in \mathbb{R}^{6}$ and the disturbance vector $\tau_{d}^{*} \in \mathbb{R}^{3}$ respectively.

In order to facilitate the subsequent development, a filtered tracking error $r(t) \in \mathbb{R}^{3}$ is expressed as follows:

$$
r \triangleq \dot{e}_{v}+\alpha e_{v}
$$

where $\alpha \in \mathbb{R}^{3 \times 3}$ is a positive-definite, constant, diagonal control gain matrix.

\section{A. Robust Control Design}

Based on the open-loop error system dynamics given in Equation (35) and the subsequent stability analysis, the control torque input is designed as follows:

$$
\tau^{*}=-\hat{F}-K r-v_{r}-\frac{e_{v}}{\left(1-e_{v}^{T} e_{v}\right)^{2}}
$$

where $K \in \mathbb{R}^{3 \times 3}$ is a constant, positive-definite, diagonal control gain matrix and $\hat{F}\left(e_{v}, e_{0}, \dot{e}_{v}, \omega_{d}, \dot{\omega}_{d}\right) \in \mathbb{R}^{3}$ is defined as

$$
\widehat{F} \triangleq Y(\cdot) \hat{\theta}+\hat{\tau}_{d}^{*}
$$

The first term $Y\left(e_{v}, e_{0}, \dot{e}_{v}, \omega_{d}, \dot{\omega}_{d}\right) \hat{\theta}$ is formed based on the following parameterization

$$
Y(\cdot) \theta \triangleq J^{*} \alpha \dot{e}_{v}+C^{*} \alpha e_{v}-N^{*}
$$

Hence, the resulting mismatch term can be defined as

$$
\tilde{F} \triangleq F-\hat{F}=Y \tilde{\theta}+\tilde{\tau}_{d}^{*}
$$

where $\mathrm{F}$ contains the unknown vector of inertia parameters and the disturbance vector as follows:

$$
F=Y \theta+\tau_{d}^{*}
$$

Based on the boundedness assumption of the disturbance vector as indicated in Remark 1, Property 1, the boundedness property of the rotation matrixes and with the use of Equation (39), the mismatch term $\tilde{F}\left(e_{v}, e_{0}, \dot{e}_{v}, \omega_{d}, \dot{\omega}_{d}\right) \in \mathbb{R}^{3}$ can be upper bounded in the following way [22]

$$
\|\tilde{F}\| \leq \rho_{1}\left(e_{v}, e_{0}, \dot{e}_{v}, \omega_{d}, \dot{\omega}_{d}\right)+\rho_{2}(\cdot)=\rho(\cdot)
$$

where $\rho(\cdot) \in \mathbb{R}$ denotes positive bounding constant and $\rho_{1}, \rho_{2}$ are positive, scalar, known bounding functions related with the parameter estimation error and the disturbance estimation error with residuals respectively. In Equation (45), the robust term, $v_{r}(t) \in \mathbb{R}^{3}$ is designed for attenuation of the mismatch and can be defined as

$$
v_{r}=\frac{\rho^{2} r}{\rho\|r\|+\varepsilon}
$$

where $\varepsilon \in \mathbb{R}$ is an arbitrarily small positive design constant.

Taking the time derivative of Equation (44), premultiplying both sides of that equation by $J^{*}$ and substituting Equation (35) and (44) for $\dot{e}_{v}$ into the resulting equation, the following expression can be obtained

$$
J^{*} \dot{r}=-C^{*} r+F+\tau^{*}
$$

After substituting Equation (45) into Equation (52), the final closed-loop error system can be obtained in the following form:

$$
J^{*} \dot{r}=-C^{*} r+\tilde{F}-K r-v_{r}-\frac{e_{v}}{\left(1-e_{v}^{T} e_{v}\right)^{2}}
$$

where $\tilde{F}$ was defined in Equation (48).

\section{B. Stability Analysis}

Theorem1: Given the closed-loop error dynamics in Equation (53), the robust control law proposed in Equation (45) ensures uniformly ultimately bounded attitude tracking in the sense that

$$
\left\|e_{v}(t)\right\| \rightarrow \gamma_{1} \exp \left(-\gamma_{2} t\right)+\gamma_{3}, \quad \gamma_{1}, \gamma_{2}, \gamma_{3} \in \mathbb{R}
$$

provided that the initial conditions are selected to satisfy 


$$
e_{0}(0) \neq 0
$$

Proof: To prove Theorem 1, a nonnegative function $V_{1}(t) \in$ $\mathbb{R}$ is defined as follows:

$$
V_{1}=\frac{1}{2}\left[e_{v}^{T} e_{v} /\left(1-e_{v}^{T} e_{v}\right)\right]+\frac{1}{2} r^{T} J^{*} r
$$

Note that the function $V_{2}(t)$ can be upper and lower bound as

$$
\lambda_{1}\|z\|^{2} \leq V_{1}(t) \leq \lambda_{2}\|z\|^{2}
$$

where $\lambda_{1}, \lambda_{2} \in \mathbb{R}$ are known positive bounding constants and $z(t) \in \mathbb{R}^{6}$ is defined as

$$
z \triangleq\left[\begin{array}{ll}
e_{v}^{T} / \sqrt{1-e_{v}^{T} e_{v}} & r^{T}
\end{array}\right]^{T}
$$

After taking the time derivative of Equation (56), using Equations (44), (33), (53) and Property 2, the following expression for the time derivative of $V_{1}(t)$ can be obtained

$$
\dot{V}_{1}=-\frac{e_{v}^{T} \alpha e_{v}}{\left(1-e_{v}^{T} e_{v}\right)^{2}}-r^{T} K r-r^{T} v_{r}+r^{T} \tilde{F}
$$

Substituting Equation (51) into Equation (59) and with the use of the definition given in Equation (58) and the inequality given in Equation (50), the expression in Equation (59) can be upper bound

$$
\dot{V}_{1} \leq-\beta\|z\|^{2}+\rho\|r\|-\frac{\rho^{2}\|r\|^{2}}{\rho\|r\|+\varepsilon}
$$

where $\beta=\lambda_{\min }\{\alpha, K\} \in \mathbb{R}$. after mathematical manipulations, Equation (60) can be written as follows:

$$
\dot{V}_{1} \leq-\beta\|z\|^{2}+\varepsilon
$$

The inequality given in Equation (57) can be applied to lower bound $\|z\|^{2}$ as

$$
\|z\|^{2} \geq \frac{1}{\lambda_{2}} V_{1}(t)
$$

Hence, the inequality in Equation (61) can be written in the following form

$$
\dot{V}_{1}(t) \leq-\sigma V_{1}(t)+\varepsilon
$$

where $\sigma=\frac{\beta}{\lambda_{2}} \in \mathbb{R}$. The following expression can be found when linear differential inequality is solved

$$
V_{1}(t) \leq V_{1}(0) \exp (-\sigma t)+\frac{\varepsilon}{\sigma}[1-\exp (-\sigma t)]
$$

From Equations (56) and (64), it can be shown that $r(t) \in \mathcal{L}_{\infty}$. Equation (44) can be used to obtain $e_{v}(t), \dot{e}_{v}(t) \in \mathcal{L}_{\infty}$. Equations (22), (25) and (28) reveals that $\widetilde{\omega}(t) \in \mathcal{L}_{\infty}$. Equation (26) shows $\omega(t) \in \mathcal{L}_{\infty}$. Hence, it can be proved that the control signal $\tau^{*} \in \mathcal{L}_{\infty}$. Standard signal chasing arguments can be employed to show that all other signals remain bounded.

From the inequalities in Equation (57) and (64), it can be concluded that

$$
\|z\|^{2} \leq \frac{\lambda_{2}}{\lambda_{1}}\|z(0)\|^{2} \exp \{-\sigma t\}+\frac{\varepsilon}{\sigma \lambda_{1}}
$$

Hence, it can be seen from Equation (65) that the objective given in Equation (54) is achieved.

\section{ROBUST CONTROL REFORMULATION}

In this section, the goal is to improve the robustness properties against sensor contamination through the redesign of the control structure proposed in (45) and thus to improve the performance under the assumption of measurement noise, parametric uncertainties and unknown disturbances.

Notice that the first term $\hat{F}(\cdot)$ of the proposed controller introduced in Equation (45) is formulated in terms of the error terms obtained by the use of measurement values. However, it constitutes the aforementioned complications such as computational burden of on-line computation requirement and sensor contamination. Hopefully, the following control structure can reduce these drawbacks by reformulating the term $\hat{F}(\cdot)$ as a function of the desired attitude, angular velocity and acceleration terms.

Based on the open-loop dynamics in Equation (35), and the subsequent stability analysis, the control torque input is designed as follows:

$$
\tau^{*}=-\hat{F}_{d}-K r-v_{r}-\frac{e_{v}}{\left(1-e_{v}^{T} e_{v}\right)^{2}}
$$

where $K \in \mathbb{R}^{3 \times 3}$ is a constant, positive-definite, diagonal control gain matrix, the term $v_{r}(t)$ is defined as in Equation (51) with a bounding constant yet to be designed and $\widehat{F}_{d}\left(e_{v}, e_{0}, \dot{e}_{v}, \omega_{d}, \dot{\omega}_{d}\right) \in \mathbb{R}^{3}$ is defined as

$$
\widehat{F}_{d} \triangleq Y_{d}(\cdot) \hat{\theta}+\hat{\tau}_{d}^{*}
$$

The term $Y_{d}\left(\omega_{d}, \dot{\omega}_{d}\right) \hat{\theta}$ is formed based on the following parameterization

$$
Y_{d} \theta=-\frac{1}{2} J \dot{\omega}_{d}-\frac{1}{2} \omega_{d}^{\times} J \omega_{d}
$$

After substituting Equation (66) into Equation (52) and adding and subtracting the term $Y_{d} \theta$ to the right hand side of the resulting equation, the following expression for the closed-loop error system can be obtained

$$
J^{*} \dot{r}=-C^{*} r+\psi-K r-v_{r}-\frac{e_{v}}{\left(1-e_{v}^{T} e_{v}\right)^{2}}
$$

where the mismatch term $\psi\left(e_{v}, e_{0}, \dot{e}_{v}, \omega_{d}, \dot{\omega}_{d}\right) \in \mathbb{R}^{3}$ and $\tilde{Y}\left(e_{v}, e_{0}, \dot{e}_{v}, \omega_{d}, \dot{\omega}_{d}\right) \in \mathbb{R}^{3}$ are defined as follows

$$
\begin{gathered}
\psi=\tilde{Y}+Y_{d} \tilde{\theta}+\tilde{\tau}_{d}^{*} \\
\tilde{Y}=Y \theta-Y_{d} \theta
\end{gathered}
$$

Based on the same assumptions given for the inequality in Equation (50), the mismatch term $\psi\left(e_{v}, e_{0}, \dot{e}_{v}, \omega_{d}, \dot{\omega}_{d}\right) \in \mathbb{R}^{3}$ can be upper bounded in the sense that 


$$
\|\psi\| \leq \rho_{3}\left(e_{v}, e_{0}, \dot{e}_{v}, \omega_{d}, \dot{\omega}_{d}\right)+\rho_{4}(\cdot)=\rho_{5}(\cdot)
$$

where $\rho_{5}(\cdot) \in \mathbb{R}$ denotes positive bounding constant and $\rho_{3}, \rho_{4}$ are positive, scalar, known bounding functions related with the parameter estimation error and the disturbance estimation error with residuals respectively.

Theorem 2: Given the closed-loop error dynamics in Equation (69), the robust control law proposed in Equation (66) ensures uniformly ultimately bounded attitude tracking in the sense that

$$
\left\|e_{v}(t)\right\| \rightarrow \gamma_{4} \exp \left(-\gamma_{5} t\right)+\gamma_{6}, \quad \gamma_{4}, \gamma_{5}, \gamma_{6} \in \mathbb{R}
$$

provided that the initial conditions are selected to satisfy

$$
e_{0}(0) \neq 0
$$

Proof: To prove Theorem 2, nonnegative function $V_{2}(t) \in \mathbb{R}$ is defined as

$$
V_{2}=\frac{1}{2}\left[e_{v}^{T} e_{v} /\left(1-e_{v}^{T} e_{v}\right)\right]+\frac{1}{2} r^{T} J^{*} r
$$

Using similar mathematical manipulations presented in the previous section, the time derivative of the function $V_{2}(t)$ can be obtained as follows:

$$
\dot{V}_{2}=-\frac{e_{v}^{T} \alpha e_{v}}{\left(1-e_{v}^{T} e_{v}\right)^{2}}-r^{T} K r-r^{T} v_{r}+r^{T} \psi
$$

Equation (76) can be upper bounded as

$$
\dot{V}_{2} \leq-\beta\|z\|^{2}+\rho_{5}\|r\|-\frac{\rho_{5}^{2}\|r\|^{2}}{\rho_{5}\|r\|+\varepsilon}
$$

where $z(t)$ is defined in Equation (58). The above inequality can be further bounded as follows:

$$
\dot{V}_{2} \leq-\sigma V_{2}(t)+\varepsilon
$$

The solution of the resulting linear differential inequality is

$$
V_{2}(t) \leq V_{2}(0) \exp (-\sigma t)+\frac{\varepsilon}{\sigma}[1-\exp (-\sigma t)]
$$

Boundedness of the error signals and the control torque input can be shown by a similar arguments used in the previous section. From Equations (57) and (79), it can be concluded that

$$
\|z\|^{2} \leq \frac{\lambda_{2}}{\lambda_{1}}\|z(0)\|^{2} \exp \{-\sigma t\}+\frac{\varepsilon}{\sigma \lambda_{1}}
$$

Hence, the objective presented in Equation (73) is achieved.

\section{Simulation Results}

In this section, simulation results are presented to illustrate the performances of the proposed control methods presented in Equation (45) and (66). The inertia matrix of the satellite is selected as follows:

$$
J=\left[\begin{array}{lll}
20 & 1.2 & 0,9 \\
1.2 & 17 & 1.4 \\
0.9 & 1.4 & 15
\end{array}\right] \mathrm{kg} \mathrm{m}^{2}
$$

The initial attitude of the satellite is selected to be

$$
\begin{aligned}
q_{0}(0)=0.9486 & q_{v}(0) \\
& =\left[\begin{array}{lll}
0.1826 & 0.1826 & 0.1826
\end{array}\right]^{T}
\end{aligned}
$$

To have a smooth rotation, the desired trajectory was set according to the following desired angular velocity

$$
\omega_{d}=0.05\left[\begin{array}{ccc}
\cos (0.1 t) & \cos (0.1 t) & \cos (0.1 t)
\end{array}\right]^{T} \mathrm{rad}
$$

The followings are the initial values of the desired quaternion

$$
q_{0 d}(0)=1 \quad q_{v d}(0)=\left[\begin{array}{lll}
0 & 0 & 0
\end{array}\right]^{T}
$$

The external disturbances are set to be

$$
\tau_{d}=0.2[\sin (t) \sin (t) \sin (t)]^{T} \quad \mathrm{Nm}
$$

Sensor noise is assumed to be zero mean Gaussian noise with 0.004 variance. Estimates of the inertia matrix and the disturbance vector are set to

$$
\hat{J}=0.7 J \quad \hat{\tau}_{d}=0.2 \tau_{d}
$$

The followings are the values of the control gains that are tuned by trial and error until a satisfactory performance is obtained

$$
K=\alpha=\operatorname{diag}\left\{\begin{array}{lll}
5 & 5 & 5
\end{array}\right\} \quad \varepsilon=0.04 \quad \rho=\rho_{5}=7
$$




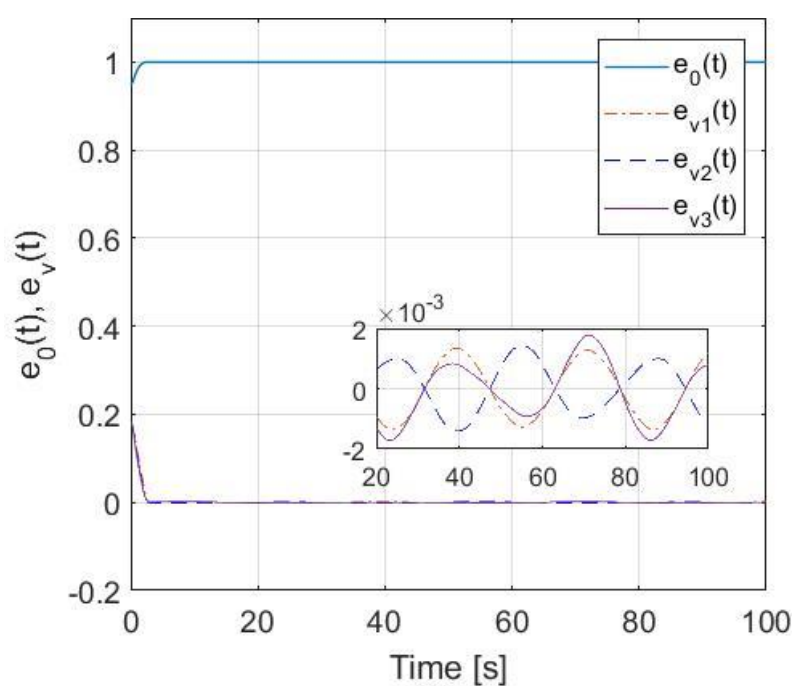

Figure 1. Controller I quaternion tracking errors (parametric uncertainties + disturbance)

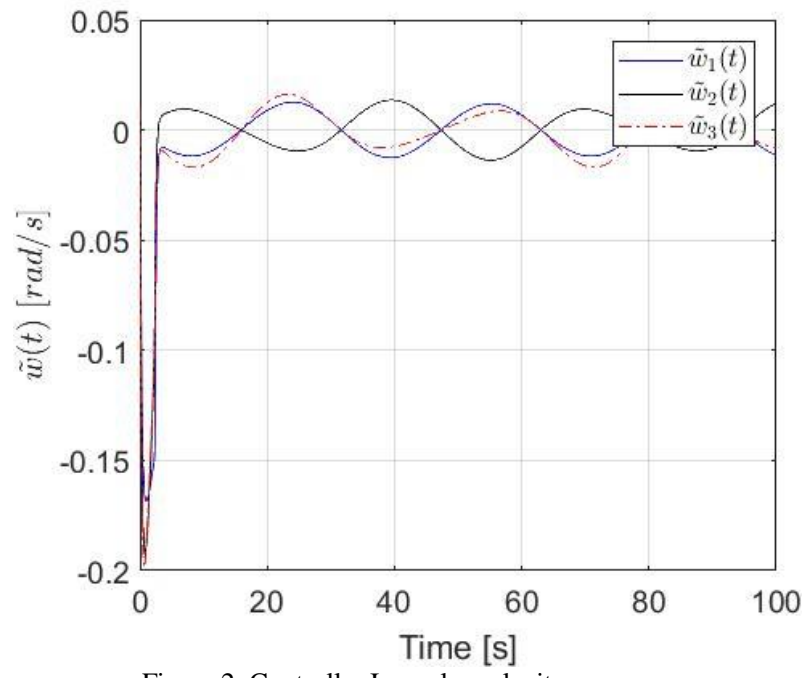

Figure 2. Controller I angular velocity errors (parametric uncertainties + disturbance)

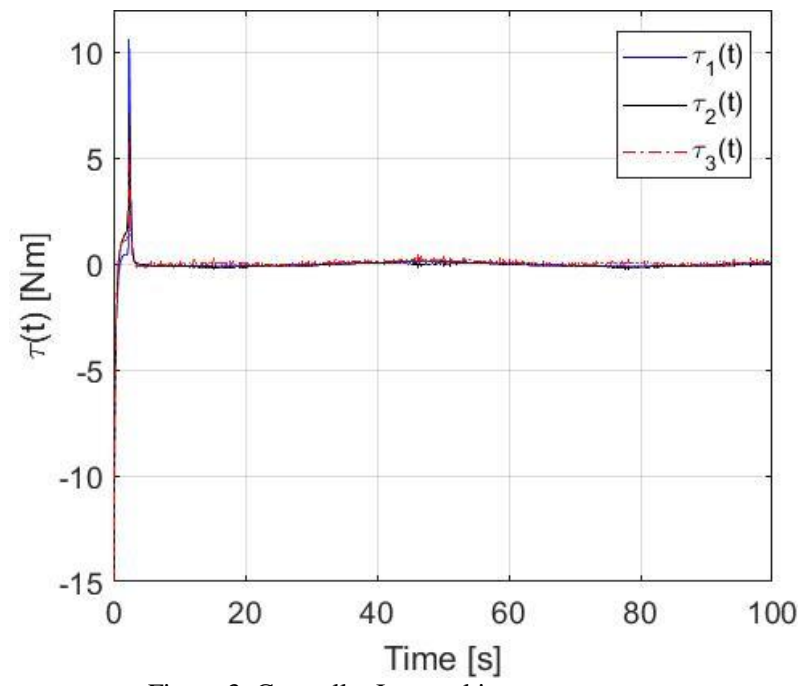

Figure 3. Controller I control input torques (parametric uncertainties + disturbance)

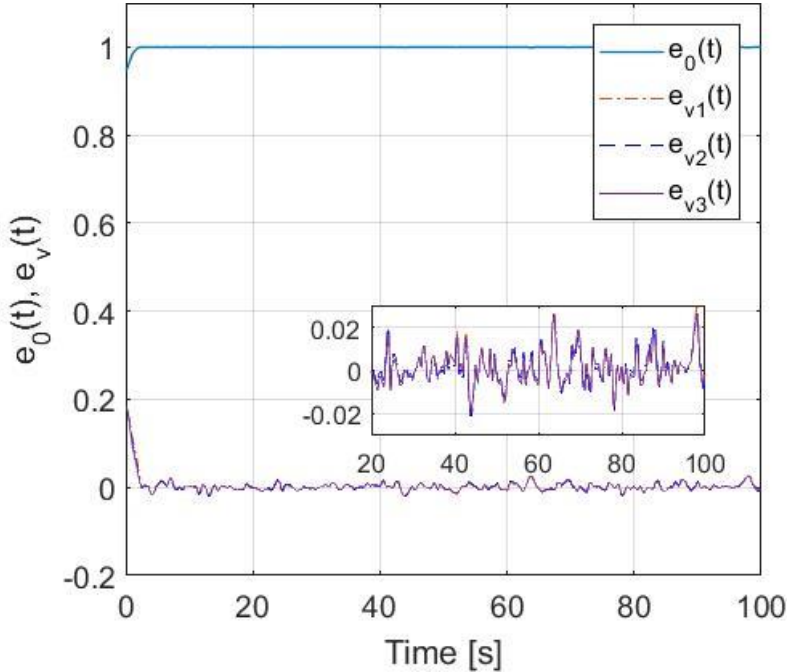

Figure 4. Controller I quaternion tracking error (parametric uncertainties+disturbance+sensor noise)

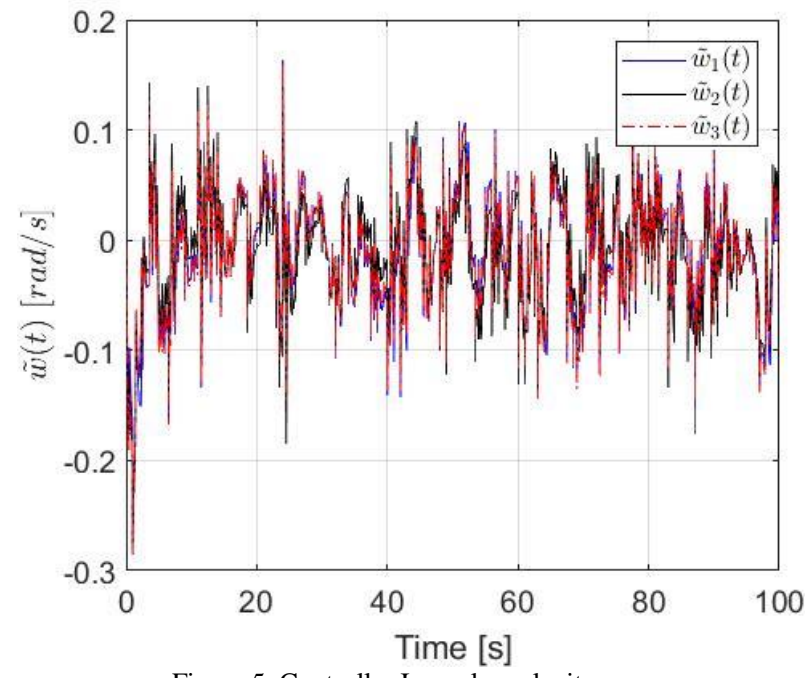

Figure 5. Controller I angular velocity errors (parametric uncertainties + disturbance + sensor noise)

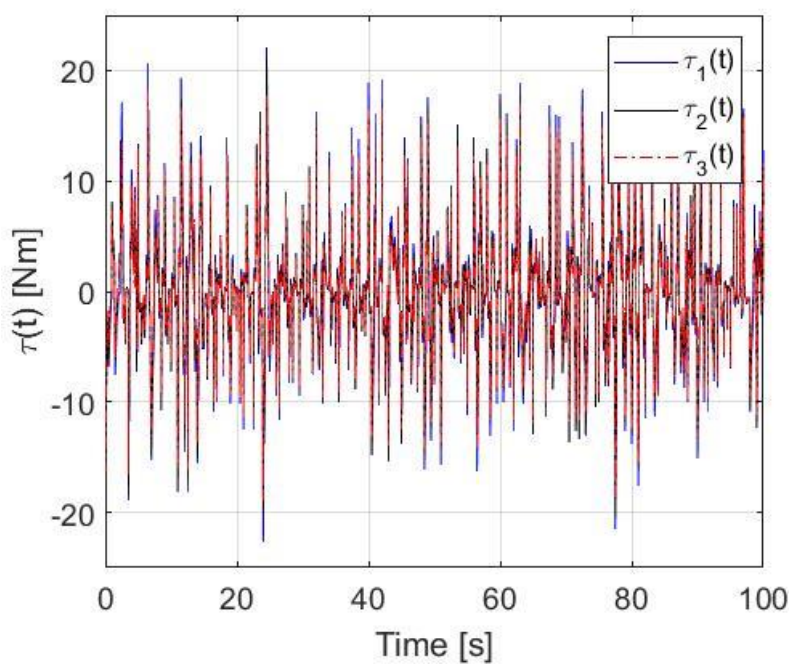

Figure 6. Controller I control input torques (parametric uncertainties + disturbance + sensor noise) 


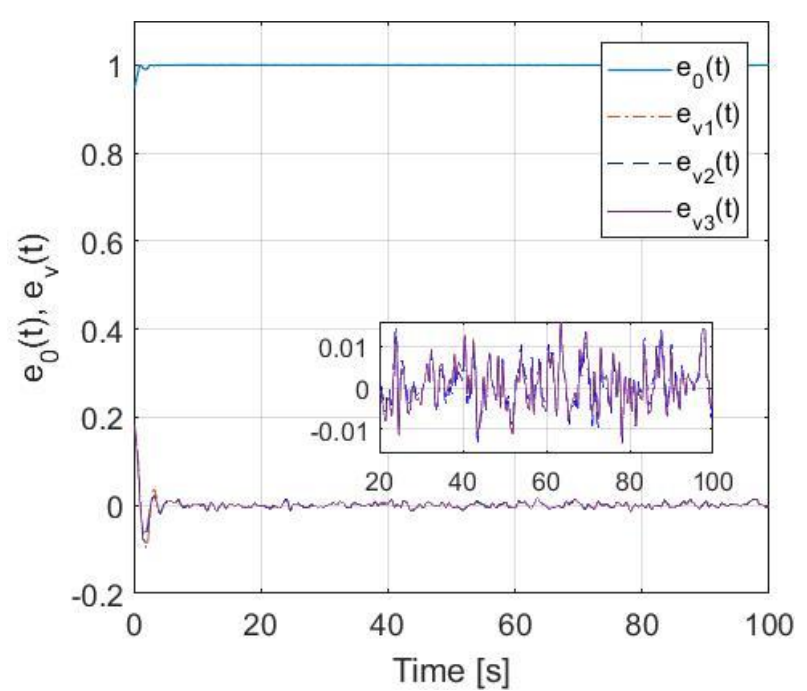

Figure 7. Controller II quaternion tracking errors (parametric uncertainties+disturbance+sensor noise)

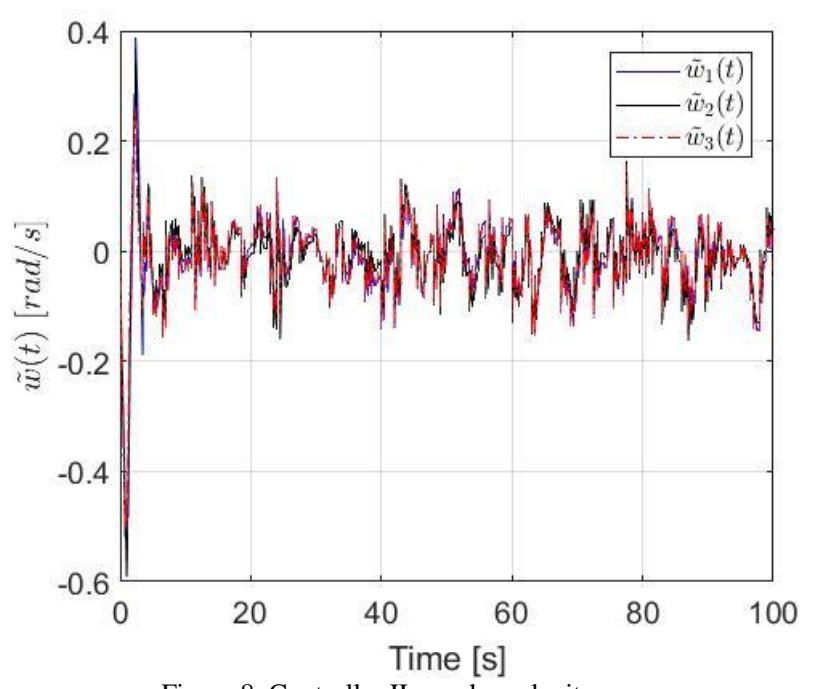

Figure 8. Controller II angular velocity errors (parametric uncertainties+disturbance+sensor noise)

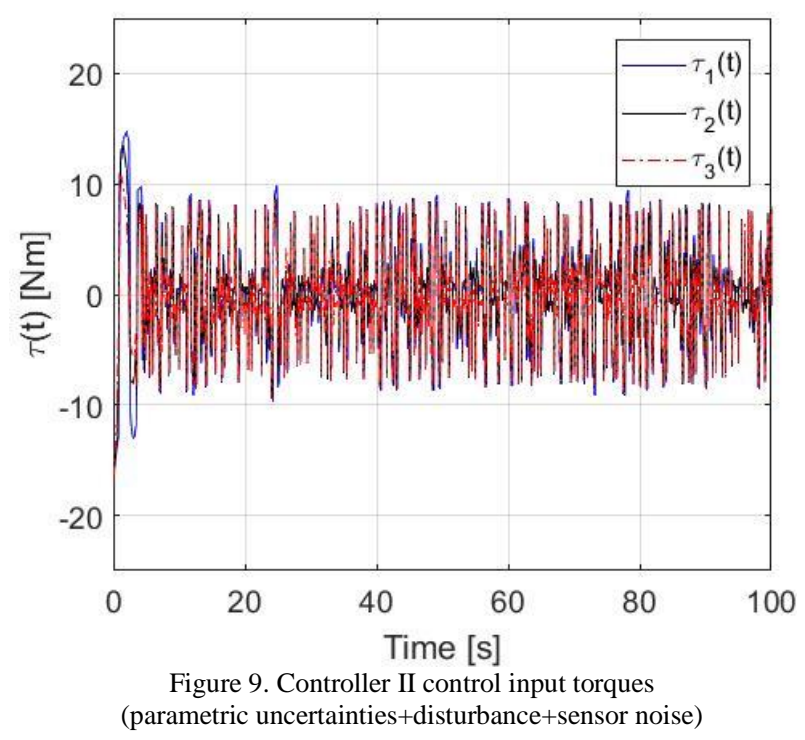

In the first part of the simulation studies, the former robust control method was examined under parametric uncertainties and disturbance effects. Fig. 1 illustrates the quaternion errors of the satellite. Angular velocity errors and the applied control input signals that belong to the same control structure are presented in Figs. 2 and 3 respectively. From Figs. 1 and 2, it can be seen that the control objective stated in the first Theorem was achieved with satisfactory performance and Fig. 3 shows that the control input signals do not exceed $0.5 \mathrm{Nm}$ after $5 \mathrm{~s}$. In the second part of the simulation studies, to compare their performances, both control algorithms were run in the presence of parametric uncertainties, external disturbance and sensor noise. Figs. 4-6 shows the results of the conventional robust controller presented earlier and Figs. 7-9 belong to the reformulated control method. From the Figs. 7-9, it was seen that the reformulated robust control method acts quite better than the conventional robust controller and both the error signals and the applied control signals were relatively reduced. During the simulation studies, it has been observed that the advantages of the second controller become more apparent in the case of increased sensor noise.

\section{CONCLUSION}

In this paper, two quaternion-based robust control method to solve the attitude tracking control problem of satellite were introduced. The first one is a conventional high-frequency robust control mechanism. It was shown that under parametric uncertainties and extern disturbance effects, it achieves well and provides uniformly ultimately bounded tracking result. However, in case of a sensor noise the controller performance was severely affected. The robust controller was then reformulated to reduce the effects of sensor contamination with less on-line computational requirement. As a future work, a new robust control mechanism can be developed to meet less sensor measurement requirements with a higher performance

\section{REFERENCES}

[1] G. Meyer, Design and Global Analysis of Spacecraft Attitude Control Systems, NASA TR R-361, March 1971.

[2] J. Y. Wen, K. Kreutz-Delgado, The Attitude Control Problem, IEEE Transactions on Automatic Control, vol. 36, no. 10, pp. 1148-1162, 1991.

[3] N. A. Chaturverdi, A. K. Sanyal, N. H. McClamroch, Rigid-body Attitude Control, IEEE Control Systems Magazine, vol. 31, no. 3, pp 30-51, 2011.

[4] M. D. Shuster, A Survey of Attitude Representations, Journal of Astronautical Sciences, vol. 41, no.4, pp.439-517, 1993.

[5] Y. Bai, J. D. Biggs, F. B. Zazzera, N. Cui, Adaptive Attitude Tracking with Active Uncertainty Rejection, Journal of Guidance, Control, and Dynamics, vol. 41, no. 2, pp. 550-558, 2018.

[6] D. Thakur, S. Srikant, M. R. Akella, Adaptive attitude-Tracking Control of Spacecraft with Uncertain Time-Varying Inertia Parameters, Journal of Guidance, Control, and Dynamics, vol. 38, no. 1, pp. 41-52, 2015.

[7] L. Cao, X. L. Chen, Y. Zhao, Minimum Sliding Mode Error Feedback Control for Fault Tolerant Small Satellite Attitude Control, Advances in Space Research, vol. 53, no.2, pp. 309-324, 2014.

[8] A. Sofyali, E. M. Jafarov, Integral Sliding Mode Control of Small Sattelite Attitude Motion by Purely Magnetic Actuation, IFAC Proc. Volumes, vol. 47, no.3, pp. 7947-7953, 2014.

[9] Z-G. Zhou, Y-A. Zhang, X-N. Shi, D. Zhou, Robust attitude tracking for Rigid Spacecraft with Prescribed Transient Performance, Int. Journal of Control, vol. 90, Iss. 11, 2017.

[10] A. Safa, M. Baradarannia, H. Kharrati, S. Khanmohammadi, Robust Attitude Tracking Control for a Rigid Spacecraft Under Input Delays and Actuator Errors, Int. Journal of Control, vol. 0, Iss. 0, 2017. 
[11] V. Utkin, J. Guldner, J. Shi, Sliding Mode Control in Electromechanical Systems, PA, Philadelphia, 1999.

[12] J. P. Hespanha, D. Liberzon, A. S. Morse, Overcoming The Limitations of Adaptive Control by means of Logic-based Switching, Syst. Contr. Lett., vol. 49, no. 1, pp. 49-65, 2003.

[13] P. A. Ioannou, J. Sun, Robust Adaptive Control, NJ, Upper Saddle River: Prentice-Hall, 1996.

[14] H. Gui, G. Vukovich, Global Finite-time Attitude Tracking via Quaternion Feedback, Syst, Contr. Lett., vol. 97, pp. 176-183, 2016.

[15] S. Wu, G. Radice, Y. Gao, Z. Sun, Quaternion-based Finite-time Control for Spacecraft Attitude Tracking, Acta Astronica, vol. 69, n0. 1, pp. 48$58,2011$.

[16] K. Lu, Y. Xia, Finit-time Fault-Tolerant Control for Rigid Spacecraft with Actuator Saturations, IET Control Theory \& Appl., vol. 7, n0. 11, pp. 1529-1539, 2013.

[17] A. Behal, W. Dixon, D. M. Dawson, B. Xian, Lyapunov-Based Control of Robotic Systems, CRC Press, 2010.

[18] M. Wood, W. H. Chen, Attitude Control of Magnetically Actuated Satellites with an uneven Inertia Distribution, Aerospace Science and Technology, vol. 25, pp. 29-39, 2013

[19] B. T. Costic, D. M. Dawson, M. S. d. Queiroz, V. Kapila, QuaternionBased Adaptive Attitude Tracking Controller Without Velocity Measurements, Journal of Guidance, Control and Dynamics, vol. 24, no. 6, pp. 1214-1222, 2001.

[20] B. Wie, Spacecraft Vehicle Dynamics and Control, AIAA, 1998.

[21] P. C. Hughes, Spacecraft Attitude Dynamics, Wiley, 1986.

[22] W. E. Dixon, A. Behal, D. M. Dawson, S. Nagarkatti, Nonlinear Control of Engineering Systems: A Lyapunov-Based Approach, MA, Boston: Birkhuser, 2003.

\section{BIOGRAPHIES}

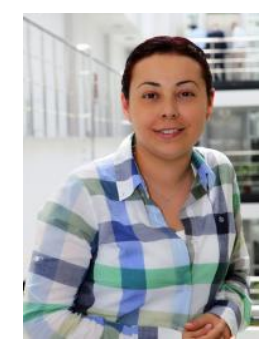

JANSET DASDEMIR, received the B.S. degree from the Electrical Engineering Department, Yildiz Technical University, Istanbul, Turkey in 2000, and the M.Sc. and Ph.D. degrees from the same university, in 2002 and 2008 respectively.

She was a Post-Doctoral Researcher at LSS-CNRS, France. She is currently involved in research with the Department of Control and Automation Engineering, YTU, Turkey, as an Assistant Professor. Her research interests include robust/adaptive control of non-linear systems, partial state feedback and output feedback control and cooperative control. 